\title{
Development Status of a 3-D Electron Fluid Model for Hall Thruster Plume Simulations
}

\author{
Maria Choi and John Yim \\ NASA Glenn Research Center, Cleveland, $\mathrm{OH}$
}

July 11, 2018

$53^{\text {rd }}$ Joint Propulsion Conference 


\section{Background}

- Simulation results are most often validated through comparisons with ground facility measurement data, while models need to predict on-orbit EP thruster operations

- Predicting behavior of flight system requires deep understanding of components and system-level interaction (e.g., thruster, cathode, facility effects, backsputtering)

- While the background pressure effects have been extensively studied in the past, ${ }^{1-6}$ we still have limited understanding electrical facility effects in vacuum chambers

- The presence of conducting chamber walls provides alternate pathways for electrons to travel from the cathode and serve as a recombination site ${ }^{7-8}$

- Need to better understand how electrons in Hall thruster plumes travel through and interact with the metallic walls of vacuum chambers 


\section{Development of an Electron Plume Model}

- In general, the Boltzmann relation is used to simulate electrons in EP thruster plumes

- Simple and useful for isothermal, collisionless, \& unmagnetized regions

- Assumes zero-electron current everywhere

- Unable to capture strong gradients in the near-field plume

- A detailed electron fluid model was developed by Boyd \& Yim using a current conservation and Generalized Ohm's law ${ }^{9-10}$

- Can predict plume characteristics better than the Boltzmann relation

- Still could not capture steep gradients in the near-field plume

- This study was further improved by including a full electron mobility tensor to account for anisotropies in electron transport due to magnetic field effects (Choi 2016)

- Similar approach in 3-D is being pursued at NASA GRC 


\section{3-D Electron Plume Model for TURF}

- The Thermophysics Universal Research Framework (TURF) is developed by Air Force Research Laboratory (AFRL)

- Many capabilities including but not limited to:

"Vlasov, particle, fluid, and hybrid simulation;

" Flexible geometry import;

"Surface interaction and charging modules ${ }^{12}$

- This electron model is currently being implemented in TURF to perform:

- Full vacuum facility simulations with magnetic field effects

- Full spacecraft integration modeling with surface charging capability 


\section{Physical Model}

- Generalized Ohm's law:

$$
\boldsymbol{j}_{e}=\mu_{e}\left(\boldsymbol{j}_{e} \times \boldsymbol{B}\right)+\sigma_{e}\left(\boldsymbol{E}+\frac{1}{e n_{e}} \nabla P_{e}\right)
$$

- Steady-state current conservation:

$$
\nabla \cdot\left(\boldsymbol{j}_{e}+\boldsymbol{j}_{i}\right)=0
$$

- Rearranging, we get:

$$
\nabla \cdot\left(\overline{\bar{\mu}}^{-1} \sigma_{e} \nabla \phi\right)=\nabla \cdot\left(\overline{\bar{\mu}}^{-1} \frac{\sigma_{e}}{e n_{e}} \nabla P_{e}\right)+\nabla \cdot \boldsymbol{j}_{i}
$$

where the electron mobility is $\mu_{e}=\frac{q}{m_{e} v_{c e}}$ and the full tensor is defined as: $\quad \overline{\bar{\mu}}=\left[\begin{array}{ccc}1 & -\mu_{e} B_{x} & \mu_{e} B_{z} \\ \mu_{e} B_{x} & 1 & -\mu_{e} B_{y} \\ -\mu_{e} B_{z} & \mu_{e} B_{y} & 1\end{array}\right]$ 


\section{Numerical Model}

- $2^{\text {nd }}$ order cell-centered finite volume approach is used

$$
\int_{V}\left[\nabla \cdot\left(\overline{\bar{\mu}}^{-1} \sigma_{e} \nabla \phi\right)\right] d V=\int_{V}\left[\nabla \cdot\left(\overline{\bar{\mu}}^{-1} \frac{\sigma_{e}}{e n_{e}} \nabla P_{e}+\boldsymbol{j}_{i}\right)\right] d V
$$

- Using the Green's theorem, the volume integral is transformed into surface integrals along all faces

- The final discretized equation becomes a linear system of equations that is solved explicitly $(A \boldsymbol{x}=\boldsymbol{b})$

- $\mathrm{FVM}=\mathrm{FDM}$ for this equation

(a)

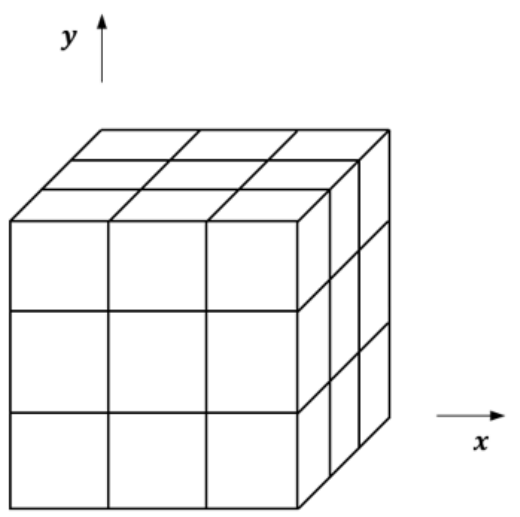

(b)

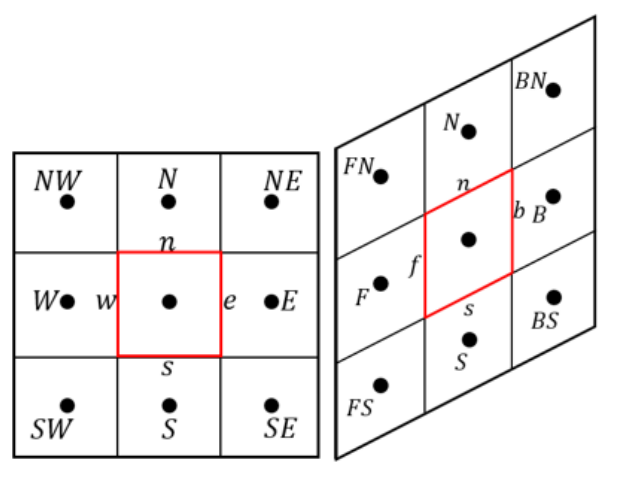




\section{Boundary Treatment: Dirichlet Boundary}

- (a) Cell-center: the simplest way -> use ghost cells

- (b) Cell-face:

- FVM: $\left.\quad \frac{\partial \phi}{\partial x}\right|_{\frac{1}{2}}=\frac{\phi_{1}-\phi_{\frac{1}{2}}}{\left(\frac{\Delta x}{2}\right)}$

- FDM: $\quad \phi_{0}=2 \phi_{\frac{1}{2}}-\phi_{1} ; \mathrm{O}(\mathrm{h})$

$$
\phi_{0}=\frac{8}{3} \phi_{\frac{1}{2}}-2 \phi_{1}+\frac{1}{3} \phi_{2} ; \mathrm{O}\left(\mathrm{h}^{2}\right)
$$

- Flux at the boundary for (b):

- $0^{\text {th }}$ order: $\left(\sigma \overline{\bar{\mu}}^{-1}\right)_{\frac{1}{2}}=\left(\sigma \overline{\bar{\mu}}^{-1}\right)_{1}$

- $\quad 2^{\text {nd }}$ order: $\left(\sigma \overline{\bar{\mu}}^{-1}\right)_{\frac{1}{2}}=\frac{3\left(\sigma \overline{\bar{\mu}}^{-1}\right)_{1}-\left(\sigma \overline{\bar{\mu}}^{-1}\right)_{2}}{2}$

(a) Cell-center

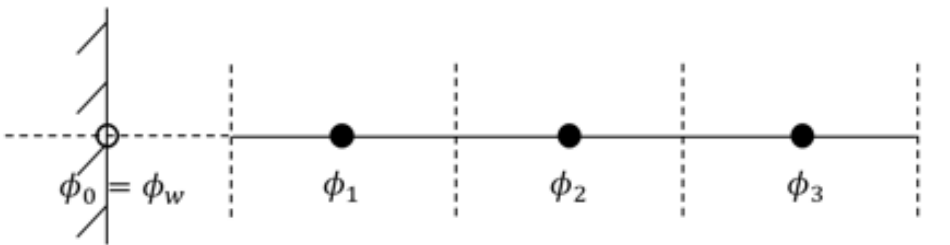

(b) Cell-face

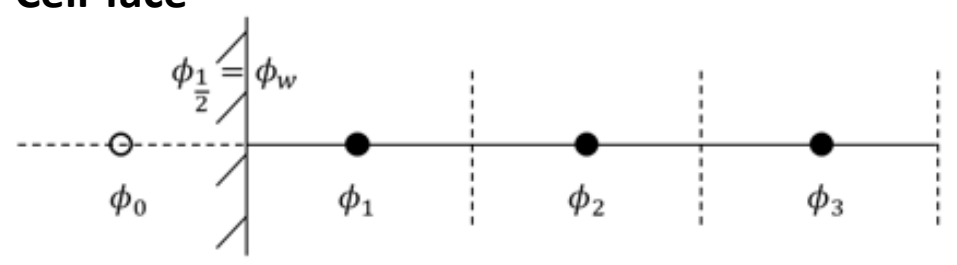




\section{Corner Boundaries (in 2-D Example)}

- Need to approximate the value of the ghost cell $\phi_{00}$

- For $\mathrm{O}(\mathrm{h})$ :

- 2-point distance-weighted averaging

$$
\phi_{00}=\frac{\phi_{10} \Delta y+\phi_{01} \Delta x}{\Delta \mathrm{x}+\Delta y} \text { which reduces to } \frac{\phi_{10}+\phi_{01}}{2} \text { for } \Delta x=\Delta y
$$

- 3-point distance-weighted averaging

$$
\phi_{00}=\frac{\phi_{10} r \Delta y+\phi_{01} r \Delta x+\phi_{11} \Delta x \Delta y}{\Delta x+\Delta y+r} \text { where } r=\sqrt{\Delta x^{2}+\Delta y^{2}}
$$

- For $\mathrm{O}\left(\mathrm{h}^{2}\right)$ :

\begin{tabular}{|c|c|c|}
\hline$\stackrel{\circ}{\phi_{02}}$ & $\dot{\phi}_{12}$ & $\dot{\phi}_{22}$ \\
\hline$\stackrel{\circ}{01}$ & $\dot{\phi}_{11}$ & $\dot{\phi}_{21}$ \\
\hline$\stackrel{\circ}{\phi 00}$ & $\stackrel{\circ}{\phi_{10}}$ & $\stackrel{\circ}{\phi_{20}}$ \\
\hline
\end{tabular}

$$
\begin{aligned}
-\phi_{00} & =\frac{\phi_{10}+\phi_{01}}{2} \\
& =\frac{1}{2}\left[\frac{8}{3}\left(\phi_{\frac{1}{2} 1}+\phi_{1 \frac{1}{2}}\right)-4 \phi_{11}+\frac{1}{3}\left(\phi_{12}+\phi_{21}\right)\right]
\end{aligned}
$$




\section{Boundary Treatment: Neumann Boundary}

- Need to calculate cell-face values

- $\phi_{0}=\phi_{1}$ for zero gradient

- $\phi_{0}=\phi_{1}+g_{w} \Delta x$ (1 $1^{\text {st }}$ order $)$ for non-zero gradient

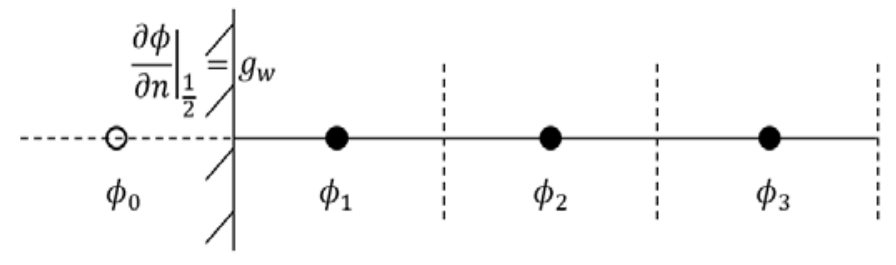




\section{Verification Tests}

- Method of Manufactured Solutions

- $\phi_{\text {exact }}=\frac{K}{6}\left(x^{2}+y^{2}+z^{2}\right)$ where $K$ is a constant

- $F=\frac{K}{3} \sigma_{e}\left(\mu_{11}+\mu_{22}+\mu_{33}\right)$

- Grid convergence study

- $\|e\|_{L_{2}}=\sqrt{\sum_{k=1}^{N_{k}} \int_{\Omega_{k}}\left(\phi_{h}-\phi_{\text {exact }}\right)^{2} d \Omega_{k}}$ 


\section{Manufactured Solution Result}

Numerical Solution

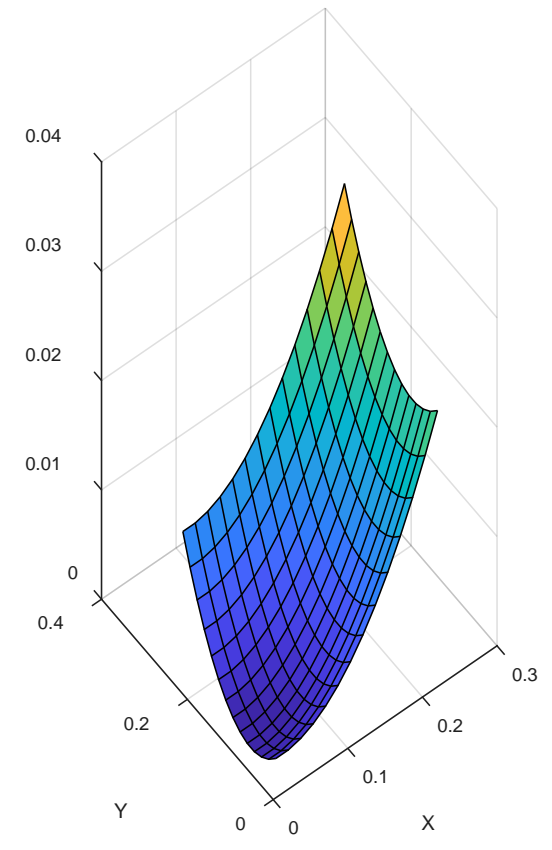

Exact Solution, $\mathrm{f}=0.5 \mathrm{x}$

$\left.{ }^{2}+y^{2}+z^{2}\right)$
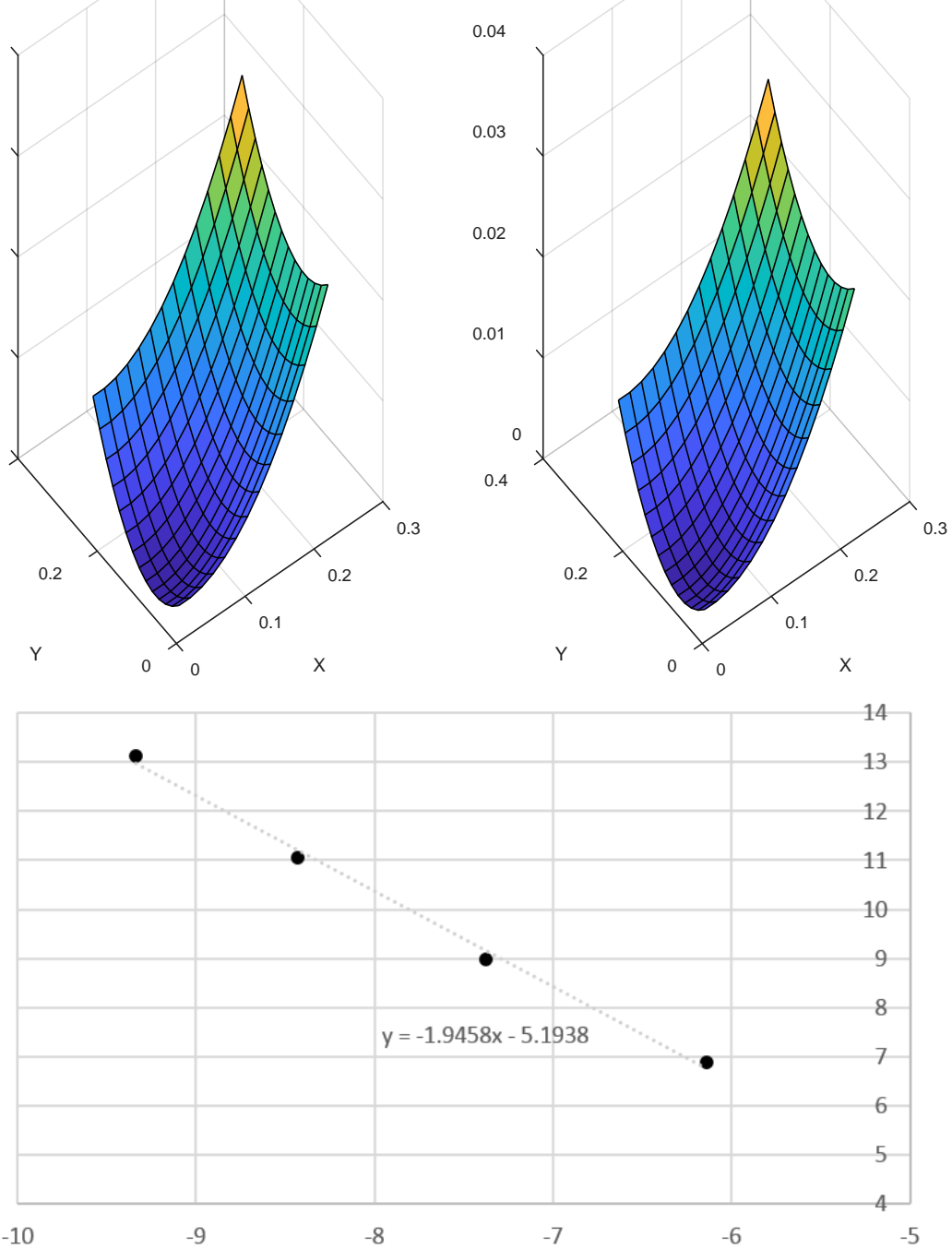

\section{3}




\section{A Hall Thruster-Like Testcase}

- Simple testcase made by Dragnea ${ }^{13}$

- Assumptions:

- $T_{e}=25 \mathrm{eV}, n_{e}=1 \mathrm{e} 17 \mathrm{~m}^{-3}$; ideal gas law

- $\boldsymbol{j}_{i}=0$

- $300 \mathrm{~V}$ on the left (anode) and $10 \mathrm{~V}$ on the right (cathode) surfaces

- Zero-flux on the top and the bottom walls

- Infinitely long plate in z-direction (periodic BC)

- Magnetic field strength as shown below:

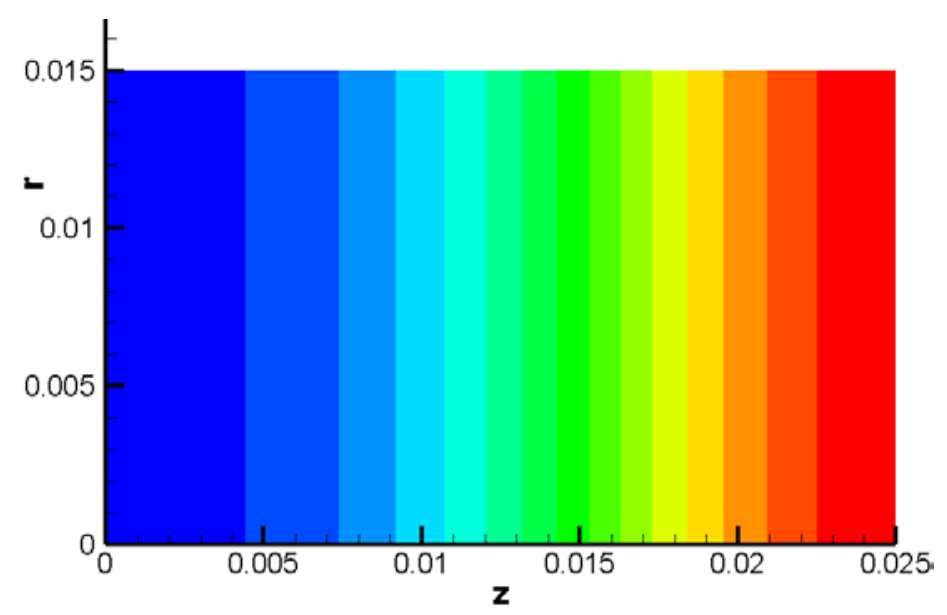




\section{Plasma Potential Calculation}

- Qualitative comparison between the 2-D axisymmetric finite element model (left) and the 3-D finite volume model (right)
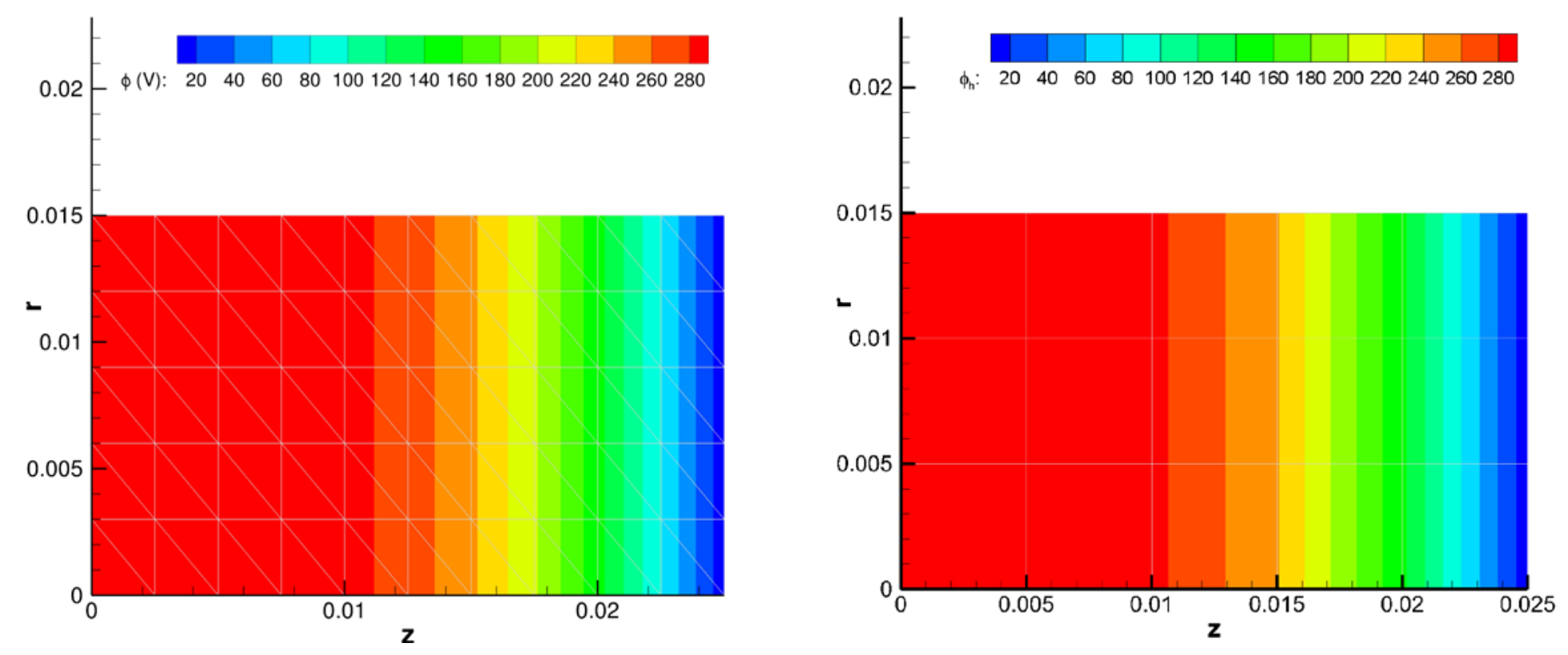


\section{Electron Current Flow Calculation}

- Use the plasma potential gradient in Generalized Ohm's law:

$$
\boldsymbol{j}_{e}=\overline{\bar{\mu}}^{-1} \sigma_{e}\left(-\nabla \phi+\frac{1}{e n_{e}} \nabla P_{e}\right)
$$

- Electron current density streamline $\left(j_{e_{z}}, j_{e_{r}}\right)$ :

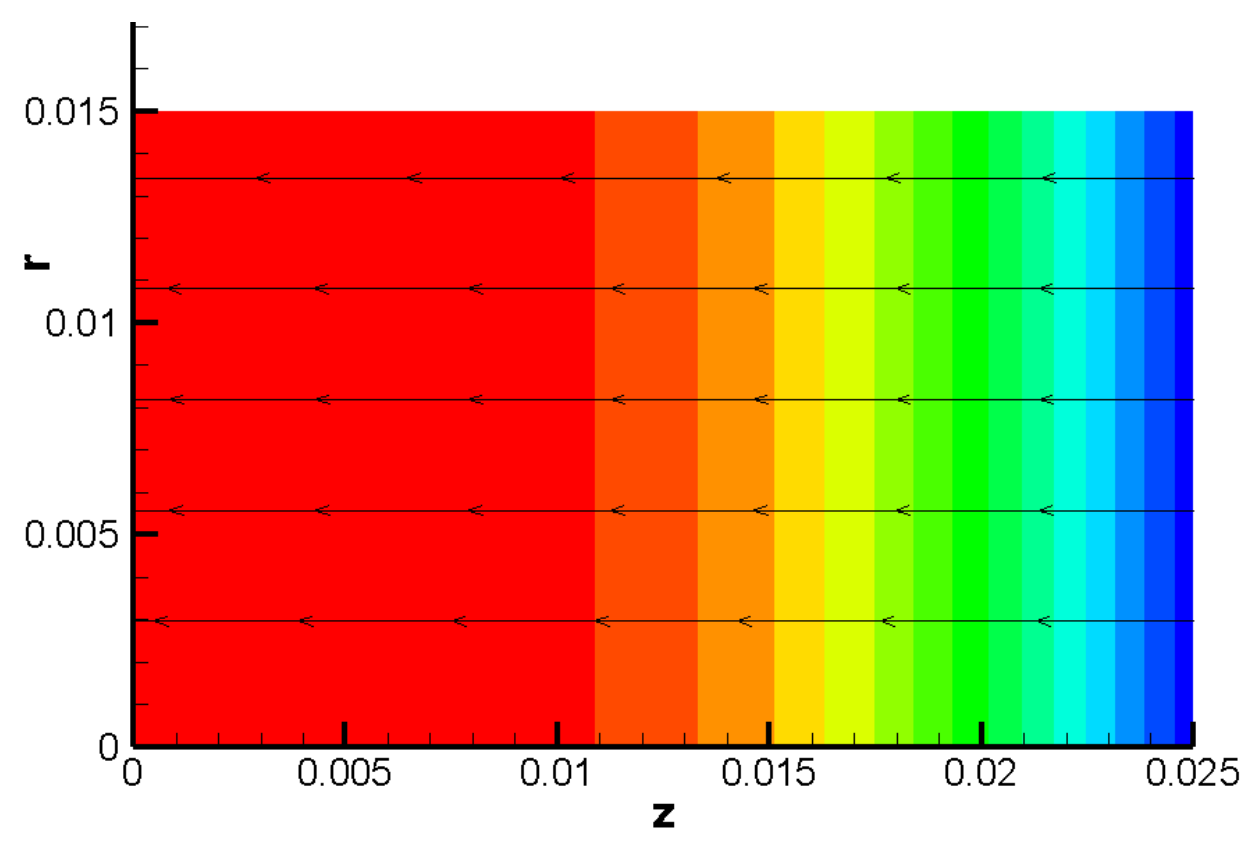




\section{Summary \& Future Work}

- A 3-D electron fluid model has been developed as a stepping stone to simulate

- the electrical facility effects in a conducting vacuum chamber

- predict electron flux values to solar arrays and other components of a spacecraft

- The model is also being implemented in TURF in collaboration with AFRL 